\title{
Analysis on the Application of Cartoon Characters in Brand Image Design
}

\author{
Xin Wei ${ }^{1, *}$ Shuai Zhang ${ }^{1}$ \\ ${ }^{1}$ College of Art and Design, Wuhan Textile University, Wuhan, Hubei, China \\ *Corresponding author. Email: 272717653@qq.com
}

\begin{abstract}
Cartoon character image refers to the portrayal of character image in animation or cartoon. When the application of animation character image in the brand image design, the brand image will leave a deep impression in the minds of consumers, and the brand influence will be enhanced. Among these institutions or enterprises that design their own images in cartoon characters, their biggest purpose is to cater to the young market and attract the attention of target consumers. It is just as why pop culture can quickly become popular among young people. Anthropomorphic products are more affinity, interesting and creative than traditional symbols or their own symbols. To explore the research of cartoon characters in brand image design from the perspective of image narration, this paper uses the method of data collection and case analysis to analyze and study the image of cartoon characters, and summarizes the application advantages of cartoon characters in brand image design. The cartoon character image makes the brand image design vivid, full of affinity, easy to understand by consumers, and has a promoting role in brand image promotion. According to the characteristics of cartoon characters, this paper provides an executable method for brand image design through the study of brand image design.
\end{abstract}

\section{Keywords: cartoon character image, brand image, personification}

\section{INTRODUCTION}

With the prosperity of material civilization, consumers pay more and more attention to spiritual consumption. Therefore, the brand image of products is becoming more and more important. Nowadays, there are many kinds of brand images, among which personification as a technique is deeply loved by consumers. The development of personification is also very mature. And the application of animation characters in brand image design has gradually become a trend, which is also used in the design and promotion of various brand images.

\section{THE APPLICATION OF PERSONIFICATION IMAGE DESIGN IN BRAND IMAGE}

\section{A. Image and setting characteristics}

Anthropomorphic image design has strong entertainment, can attract the attention of consumers, so it is widely used in a variety of visual images. The personification marketing is to humanize the brand, and obtain a variety of brand personality, so that the brand can effectively interact with the target consumer groups. Anthropomorphic design can establish a solid relationship between brand and consumer. The ideal personification marketing can establish a bridge between consumers and brands, thus forming brand attachment. With the development of the Internet, a variety of advertising and publicity penetrate deeply into people's lives. Images, audio, text and video advertisements have been bombarding consumers' senses for a long time, in a wide range of fields and in many directions. The purchasing choices made by consumers have been seriously affected. Under such information bombing, consumers have established a variety of psychological defense mechanisms, and have strong resistance to the purchase persuasion of some traditional advertisements. This kind of psychological defense mechanism weakens the marketing communication effect of the brand. Therefore, how to skillfully break through the psychological defense mechanism of consumers has become the primary goal of personification design. The application of personification in brand image can interact with consumers in a more acceptable way, and skillfully break through this psychological defense mechanism.

In the animation works, some animation designers will exaggerate the character image, so as to highlight the theme and character image of the work, enrich the story content, better show the story plot, and enhance the appeal of the story. [1] 
Brand personification will not only affect the attitude and behavior of consumers, but also affect brand equity, brand image and brand personality. [2] Personification image has been used for a long time. For example, as early as 1895, Michelin tire launched the image "Bibendum", which looked like a humanoid with inflatable tires stacked on top of one another. So far, "Bibendum" has developed into a classic Michelin logo. (as shown in "Fig. 1").

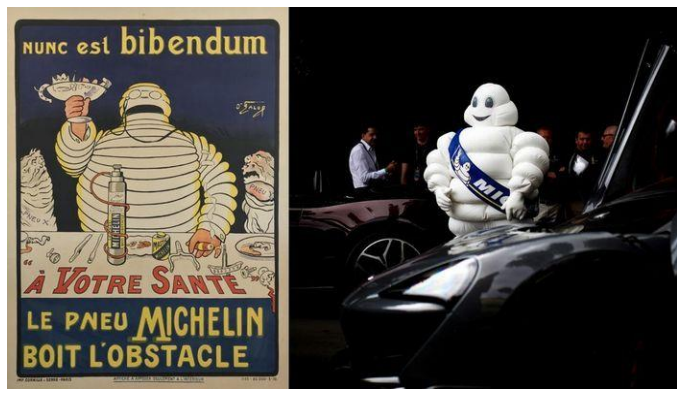

Fig. 1. "Bibendum".

The application of personification image is not only in the field of tires, but also in FMCG brands, such as, the laughing boy in "Want Want Children's Milk", the lively and lovely squirrel in "Three Squirrels", and the literary and artistic youth image in "Jiangxiaobai", etc. Brand creates differentiated functional value and emotional value for consumer groups. Consumers often pay attention to whether the emotional value of the brand can provide emotional identity claim and group belonging proposition when choosing products. For example, "Jiang Xiaobai" provides the identity proposition of literary and artistic youth. This idea endows the brand personality of "literature and art" and focuses on the market of intellectual youth groups. (see "Fig. 2", "Fig. 3", "Fig. 4").

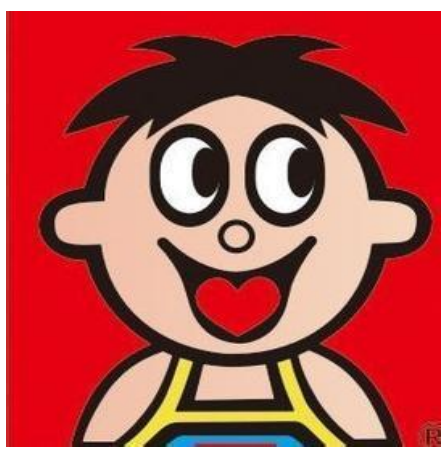

Fig. 2. Want Want Children's Milk.

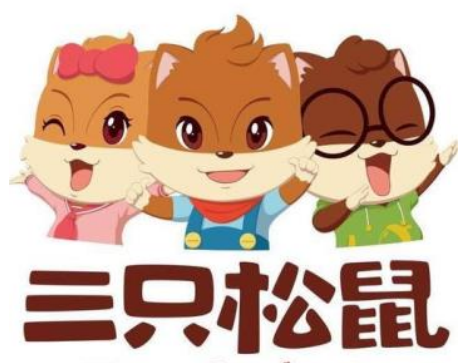

Three Squirrels

Fig. 3. Three Squirrels.

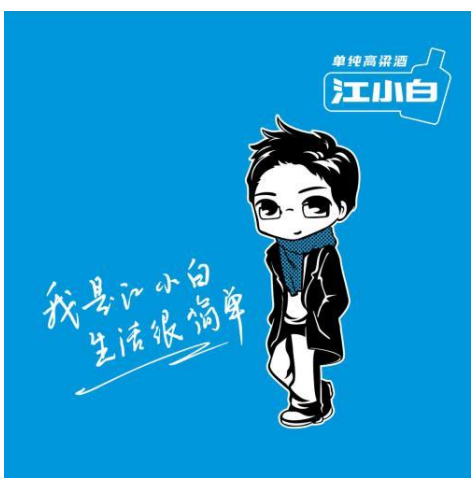

Fig. 4. "Jiangxiaobai".

\section{B. The use of cartoon characters in brand image}

People are the sum of social relations. In brand communication, consumers are often labeled with various labels and divided into different user images. When some user groups use a certain brand product and form a certain scale, some personality behavior characteristics of the group will become the example of brand personality.

With the development of today's society and the growth of the younger generation, the new generation of consumer groups has gradually grown. As Japanese animation enters the Chinese market and Chinese animation has a profound impact on the new generation of consumer groups, the image of animation characters is becoming more and more popular. The degree of anthropomorphic image has been developed further.

Every company has an image. The image is the external performance of the company. The external performance is often displayed through the craftsmanship, advertising art, brand effect, and cultural artworks, etc. [3]

For example, "BLING" of CCTV Dream Channel, "2233 Up of Bilibili, etc. directly design cartoon characters as mascots and corporate logos, rather than merely personalizing animals or still life. When the characteristics are the same as the anthropomorphic 
character, consumers are more likely to regard products and brands as human beings, and consumers' favorability will increase accordingly. [4]

The personification images usually perform a twohead "Q" version or the Japanese and Korean animation style. The character shaping lines are smooth, and the body is full of flexibility. The detailed design of the role will also be interspersed with the logo or unique representation of the enterprise or organization. Most of the characters are young men and girls with rich expressions, giving people a feeling of vigor and vitality. Brand cartoon image as a virtual salesman, its primary responsibility is to promote their own corporate brand. [5]

There is an example of the "2233" setting of "bilibilibili". Brand personification can promote consumers to participate in value co-creation more effectively than non-personification. However, when the consistency of brand values is low, there is no significant difference between brand personification and dehumanization on consumers' value co-creation intention. [6] The role is set as a girl, which has a strong attraction for its main audience group (post-90s to post00s), that is, the target consumer group. Young people's understanding of the enterprise starts from these images. After they have a basic understanding of the enterprise and its products, they become consumers of the enterprise products, and the relationship between the brand and the consumer is established. This model skillfully avoids the psychological defense of the target population. The humanized corporate image can be applied to various cultural and creative products after re-creation. The humanized corporate image itself has become a part of corporate cultural products, bringing additional benefits to the enterprise. ("Fig. 5" and "Fig. 6"). Brand character image design should be based on the brand spirit and cultural concept, and fully combine the basic elements of the brand visual identification system for creative design, so as to make the audience see the character image conform to the cultural concept or brand spirit that the brand wants to convey. [7]

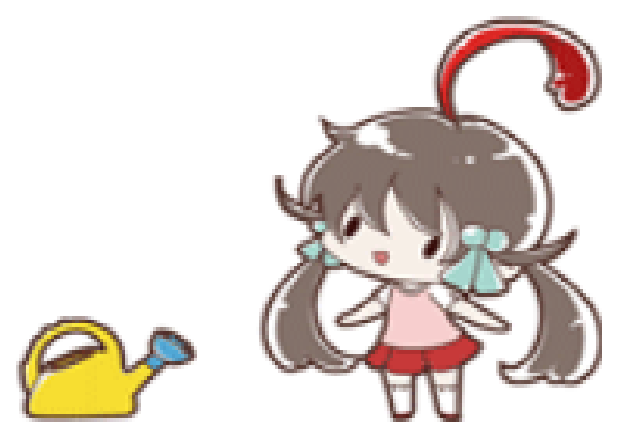

Fig. 5. "BLING" of CCTV Dream.

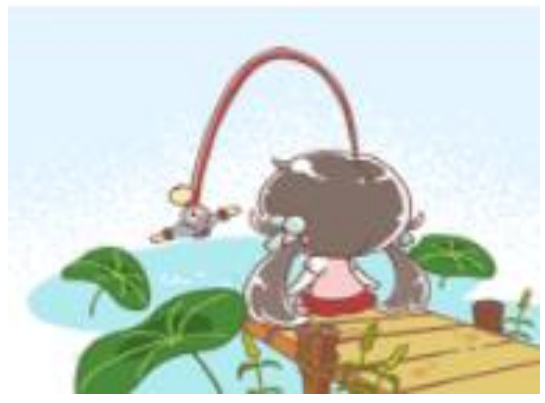

Fig. 6. "BLING" meme.

The above picture is "BLING" of CCTV Dream channel. The character performs a two-headed "Q" version. The action and manner of the characters are very vivid and lovely. "BLING" of CCTV Dream is also set as the channel's mascot and spokesperson.

In the use of color, being bright, colorful, fresh is the main melody of the screen color. For example, sky blue, lemon yellow, bright red, etc. However, in order to show the characteristics of the enterprise itself, it is very convenient to integrate the enterprise standard color into the role design.

\section{THE NEEDS OF TARGET GROUPS AND THE DEVELOPMENT OF NEW MEDIA}

\section{A. Reasons for the wide application of animation image}

Among these institutions or enterprises that design their own images in cartoon characters, their biggest purpose is to cater to the young market and attract the attention of target consumers. It is just as why pop culture can quickly become popular among young people. Compared with traditional signs or their own symbols, anthropomorphic products have more affinity, interest and recreation. There is also a more important reason. The shaping of corporate brand can not only be completed by its own design department, but also can be more personalized by consumers, so as to make the brand image have a deeper level of influence.

The "post-90s" and "post-00s" have become the main groups of the younger generation (i.e. the new generation of consumer groups). They have their own characteristics in terms of consumption concept. They have open minds and pursue fashion. Under the influence of such a concept, they are more likely to accept and more actively accept the change of corporate image. The influences of hedonism culture "amusing to death" and luxury culture with the theme of "entry lux" have promoted young people's desire to pay for personification of corporate image. This situation also provides a strong driving force for the development of corporate image personification towards more levels and more directions. 
Research shows that young people born in the 1990s to the beginning of the 21 st century have formed four action principles of happiness, rationality, experience and consumption in the process of constructing individual cultural circle and practicing their own life goals. These principles will be important reasons for their consumption culture orientation of "emphasizing form over content". Therefore, the virtual role representing the corporate image should have enough changes. There should have enough images in various festivals, events and activities. For example, during the Spring Festival, the role form should have the Spring Festival element. The vitality of this role should closely keep pace with the times and grasp hot events, and has close interaction with consumers in hot spots. The virtuality of animation characters and the close interaction with consumers will greatly enhance the brand emotional cohesion between animation image and target audience.

It can be seen that the personification of "bilibilibili" meets the aesthetic requirements of young people in various details. For example, the image and painting style of girls with bright but not gorgeous colors.

\section{B. Application of cartoon character image in brand communication}

With the birth of a series of new technologies, new materials and new expression methods have been promoted. In the aspect of anthropomorphic image building, with the birth of holographic projection technology, the plane image is presented in the form of $3 \mathrm{D}$, which elevates the display of product image to a new height. People can feel the existence of product image from many perspectives. From single-sided and static posters to omni-directional, wide perspective, multi-dimensional image, the personification of corporate image will have a broader prospect.

On the other hand, the development of new media will transfer the personified corporate image to consumers more quickly, comprehensively and carefully. For example, with the influence of WeChat official account, personification images can be transmitted through desktop wallpaper, expression, video and other channels. The humanized image can be applied to various media with its highly flexible combination method.

Brand communication of enterprises often takes brand positioning as the core, and establishes brand image with unique personality in the minds of consumers through brand contact points. Therefore, all behaviors around brand communication should be based on the premise of maintaining brand personality with high identification. For example, the image of "2233" on "bilibilibili" is consistent with its corporate image, such as the small TV image. The personality image of the spokesperson has a great influence on the brand personality. "Bilibilibili" was originally a community website for animation lovers to communicate with each other. The brand image expressed by "bilibilibili" should be consistent with the personality of its audience. Only in this way can consumers' accurate cognition of brand personality be effectively improved, and the personal style of the spokesperson can be associated with the brand personality. Therefore, it is reasonable to apply cartoon characters to brand image design.

Generally, the personification image can be divided into two types: the ability type and the warm type. The ability type of images can give people a sense of security, such as the image of Mr. Wittman. The warm type has more affinity and interest. There is an example of the image of Want Want Children's Milk. The cartoon character images are often warm. Because the animation character image takes the route of "wins by the type". The consumption of hedonic products is often dominated by perceptual consumption. Consumers have the consumption in order to meet the spiritual needs, so it is more closely related to the warm type. Warm images can also give positive brand attitude. The use of animation characters does not need to be entangled in the choice of ability type or warm type.

\section{THE APPLICATION OF CARTOON CHARACTERS IN BRAND IMAGE DESIGN}

The demand theorem proposed by psychologist Abraham Maslow points out that there are six levels of human needs, and the high-level needs are social needs, respect needs and self needs. The Internet connects everyone closely together, but this connection is based on personal mobile terminals. This connection creates new connections but also creates new obstacles. People's face-to-face communication is relatively reduced, but people's social needs are more and more urgent. People also need a medium to fulfill their social needs. Bilibili uses the cartoon image "2233" as the media to attract consumers "Bilibili users" and "animation lovers" in a circle, where consumers can realize their social needs. And the consumers in this circle will precipitate into loyal users of "bilibilibili" network, providing additional markets.

The output of animation character image is not completely decided by the enterprise design department. In order to design the image that meets the needs of consumers, the enterprise design department will not work behind closed doors, but will give the right of choice to the society, and consumers will make or vote for the image. In this way, consumers will have a sense of intimacy for the cartoon character image, which is not from the design of the animation image, but from the sense of participation and achievement of participating in the image design. When consumers 
consume such products, they not only get the actual products, but also get the experience of participating in the design. Before the brand launched the cartoon character image, it has been preheated in people's mind for its image. This method is not used for the first time in anthropomorphic image design. Consumers do not need to master professional design knowledge, only need to choose according to their own preferences. In this process, enterprises gain more attention and users, and the most important thing is to make their corporate image more perfect and full. After the personification of corporate image, the relationship between enterprises and consumers changes from the relationship between "things and people" to "people to people". Birds of a feather flock together. Successful animation image design can highlight brand personality, enhance loyalty and brand stickiness of target consumer groups.

Jiangxiaobai has created the image of "literary loser youth" and used new media to infiltrate the consumption and life scenes of consumers. Jiangxiaobai has become a phenomenal topic. Its innovative expression bottle and content of young people loving culture and arts make Jiangxiaobai's influence equal to the first-line liquor brands, but its sales volume is far less than the general liquor brands. [8]

The network animation image "2233" of "bilibilibili" also runs well. "2233" of "bilibilibili" even had her own channel, which gradually developed into an "ip". It is to promote the means of brand communication to gradually become a cultural product, which is enough to prove the broad prospects of cartoon characters in brand image design.

\section{CONCLUSION}

The application of cartoon character in brand image still needs to consider the needs of target groups. Cartoon characters are not acceptable to all age groups. The design department should be targeted rather than blindly following the trend. Even the cartoon character image should also pay attention to the difference of brand image. Otherwise, it will also fall into the problem of brand homogeneity. The recognition and difference of cartoon characters still need to be paid attention to.

\section{References}

[1] Zhang Chenhao. Research on character image design in modern animation [J]. Art Education Research, 2020(03): 62-63. (in Chinese)

[2] Wang Xiao. Research on the influence of brand personification on the purchase intention of different generations of consumers [D]. Shandong University, 2020. (in Chinese)

[3] Yang Hui. The role of cultural art in shaping corporate image [J]. Corporation Research, 2018(12): 52-54. (in Chinese)
[4] Peng Quanquan. Research on the Influence of Anthropomorphic Image on Consumers' Choices of Pleasure and Utility [D] Shanghai International Studies University, 2018. (in Chinese)

[5] Jin Jing. Corporate Brand Cartoon Image Study of the "Internet+" [D]. East China University of Science and Technology, 2019. (in Chinese)

[6] Zhu Liangjie, He Jiaxun, Huang Haiyang. The Moderating Effect of Brand Value Consistency on Consumer Value CoCreation Intention [J]. Chinese Journal of Management, 2018, 15(08): 1196-1204. (in Chinese)

[7] Zhou Xin. Anthropomorphic Design Performance of Character Image in Brand Visual Identity System [J]. Journal of Minjiang University, 2019, 40(06): 80-85. (in Chinese)

[8] Liu Jinxing, Chen Yirong. Effect of Personification Marketing on Brand Attachment [J]. The Border Economy and Culture, 2018(11): 13-15. (in Chinese) 